\title{
Low Loss Antiresonant Hollow Core Fibres
}

\author{
F. Poletti, J. R. Hayes, D. J. Richardson \\ Optoelectronics Research Centre, University of Southampton, Southampton SO17 1BJ, United Kingdom \\ frap@orc.soton.ac.uk
}

\begin{abstract}
We study the loss mechanisms in novel antiresonant hollow-core fibres and demonstrate the importance of optimising the air-cladding thickness and reducing the node size. Based on these rules we fabricate fibres with wide-bandwidth and low-loss. OCIS codes: (060.2280) Fiber design and fabrication; (060.4005) Microstructured fibers;
\end{abstract}

\section{Introduction}

Fibres that guide light in a large hollow core and in a few spatial modes are becoming increasingly important in the delivery of multi-MW peak power beams and to exploit nonlinear dynamics in gases. For high peak power beam delivery, a large air core is required to prevent fibre damage and catastrophic self-focusing effects. Hollow core Kagome'-type fibres are ideally suited for these applications but they do present an extended complex-to-make cladding [1]. Simplified hollow core fibre types guiding by antiresonance, made of a single ring of holes around the core and presenting low loss for device applications were recently reported [2-4]. Here we study loss mechanisms in these fibres and present an improved fibre design with a minimum loss below $1 \mathrm{~dB} / \mathrm{m}$ in the near infrared.

\section{Fibre design, fabrication and characterisation}

Studying the loss of circularly symmetric air-glass Bragg fibres provides good insights into the guidance of more realistic structures. It can be shown that an annular glass ring contained within a larger solid cladding has a $\lambda^{4} / \mathrm{r}^{5} \operatorname{loss}$ dependence, as compared to the $\lambda^{2} / r^{3}$ of more conventional circular hollow core fibres, and therefore can achieve the same loss in a smaller core structure, less prone to intermodal effects and bending losses. The overall loss can be minimised by imposing a quarter wave stack condition to the outer air layer, which requires its thickness to be $65 \%$ of the core radius. Besides radial resonances resulting in strong loss peaks at those wavelengths where resonance occurs in the glass core ring surround, unavoidable azimuthal resonances, lower in magnitude but denser in the spectrum, also occur in any real structure. We conducted a thorough analysis of several realistic fibres with the same core size and found that one particular design, corresponding to a hexagram or Star-of-David (SoD) shape provided consistently lower losses than any other structure studied (Fig.1a). A number of hexagram fibres were fabricated and characterised, which highlighted the importance of reducing the node size (Fig1b and 1c).

The best fibre fabricated to date has a core of $38 \mu \mathrm{m}$, average losses of around $4 \mathrm{~dB} / \mathrm{m}$ in the $800-1600 \mathrm{~nm}$ range and minimum loss below $1 \mathrm{~dB} / \mathrm{m}$ (Fig.1d). Ongoing work focuses on the understanding of the improved low-loss performance of the SoD fibre, and on means to practically reducing further the node sizes in fabricated fibres.
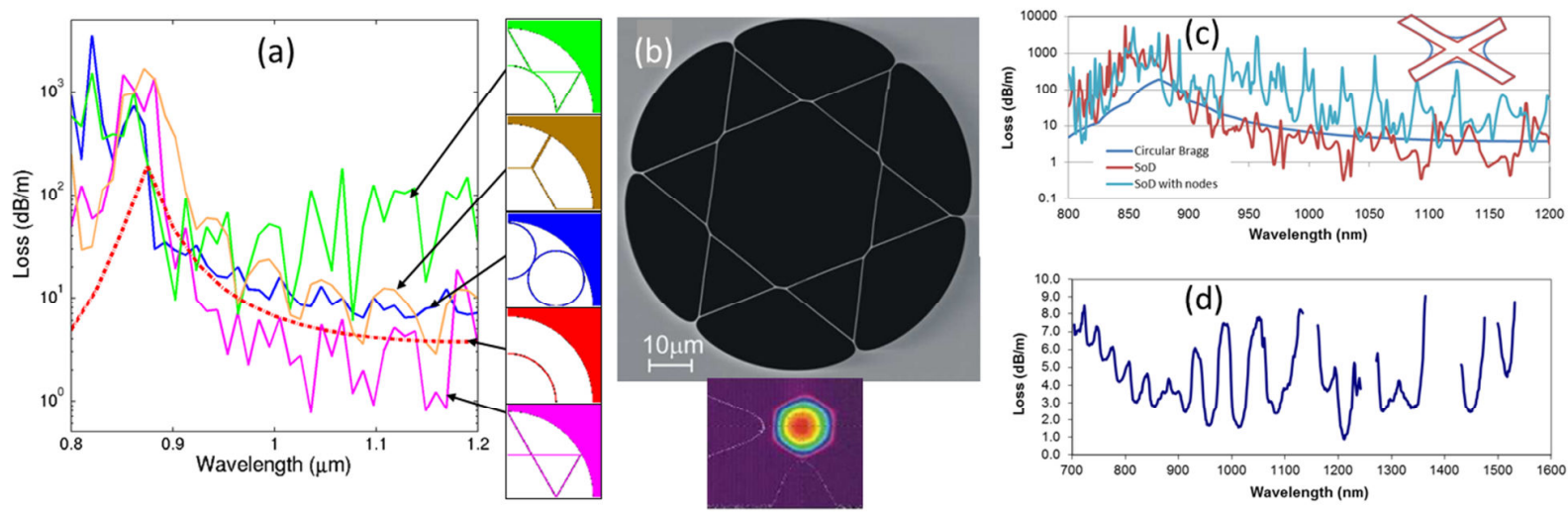

Fig. 1: (a) Simulated loss of various hollow core fibres $(r=15 \mu \mathrm{m}$, th $=420 \mathrm{~nm})$; Fabricated SoD $(r=19.8 \mu \mathrm{m}$, th $=300 \mathrm{~nm})$; $(\mathrm{c})$ calculated loss of the SoD with (blue) and without (red) nodes; (d) measured loss for the fibre in (b).

\section{References}

[1] F. Couny et al., "Generation and Photonic Guidance of Multi-Octave Optical-Frequency Combs," Science 318, 1118-1121 (2007).

[2] S. Février et al., "Understanding the origin of loss in large pitch hollow-core photonic crystal fibers," Opt. Express 18, 5142-5150 (2010).

[3] Luca Vincetti and Valerio Setti, "Waveguiding mechanism in tube lattice fibers," Opt. Express 18, 23133-23146 (2010).

[4] A. D. Pryamikov et al., "Demonstration of a waveguide regime for a silica hollow-core microstructured optical fiber with a negative curvature of the core boundary in the spectral region > $3.5 \mu \mathrm{m}$," Opt. Express 19, 1441-1448 (2011). 